\title{
Healthcare-Associated Methicillin-Resistant Staphylococcus aureus \\ Clinical characteristics and antibiotic resistance profile with emphasis on macrolide-lincosamide-streptogramin B resistance
}

"Jyoti Kumari, ${ }^{1}$ Shalini M. Shenoy, ${ }^{1}$ Shrikala Baliga, ${ }^{1}$ Chakrapani M., ${ }^{2}$ Gopalkrishna K. Bhat ${ }^{1}$

$$
\begin{aligned}
& \text { البكتيريا العنقودية الذهبية المقاومة للميثيسيلين المرتبطة بالرعلية الصحية }
\end{aligned}
$$

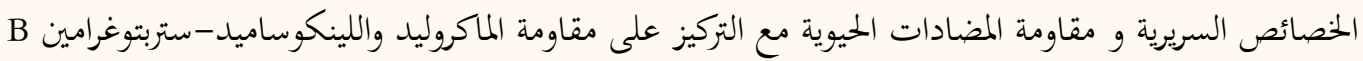

$$
\text { جيوتي كوماري، شاليني شينوي، شريكالا باليغا، شاكرباني م،، جوبالكريشنا باهت }
$$

ABSTRACT: Objectives: Healthcare-associated methicillin-resistant Staphylococcus aureus (MRSA) is a common pathogen worldwide and its multidrug resistance is a major concern. This study aimed to determine the clinical characteristics and antibiotic susceptibility profile of healthcare-associated MRSA with emphasis on resistance to macrolide-lincosamide-streptogramin B $\left(\mathrm{MLS}_{\mathrm{B}}\right)$ phenotypes and vancomycin. Methods: This cross-sectional study was carried out between February 2014 and February 2015 across four tertiary care hospitals in Mangalore, South India. Healthcare-associated infections among 291 inpatients at these hospitals were identified according to the Centers for Disease Control and Prevention guidelines. Clinical specimens were collected based on infection type. S. aureus and MRSA isolates were identified and antibiotic susceptibility tests performed using the Kirby-Bauer disk diffusion method. The minimum inhibitory concentration of vancomycin was determined using the Agar dilution method and inducible clindamycin resistance was detected with a double-disk diffusion test (D-test). Results: Out of 291 healthcare-associated S. aureus cases, 88 were MRSA (30.2\%). Of these, 54.6\% were skin and soft tissue infections. All of the isolates were susceptible to teicoplanin and linezolid. Four MRSA isolates exhibited intermediate resistance to vancomycin (4.6\%). Of the MRSA strains, 10 (11.4\%) were constitutive MLS $_{\mathrm{B}}$ phenotypes, 31 (35.2\%) were inducible $\mathrm{MLS}_{\mathrm{B}}$ phenotypes and 14 (15.9\%) were macrolide-streptogramin B phenotypes. Conclusion: Healthcare-associated MRSA multidrug resistance was alarmingly high. In routine antibiotic susceptibility testing, a D-test should always be performed if an isolate is resistant to erythromycin but susceptible to clindamycin. Determination of the minimum inhibitory concentration of vancomycin is necessary when treating patients with MRSA infections.

Keywords: Healthcare Associated Infections; Antibiotic Resistance; Methicillin-Resistant Staphylococcus aureus; Phenotypes; Clindamycin; Vancomycin.

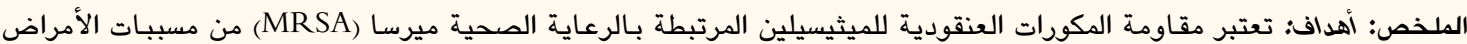

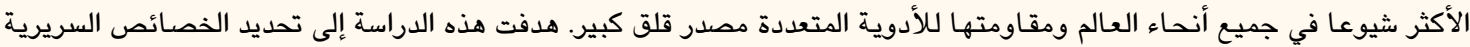

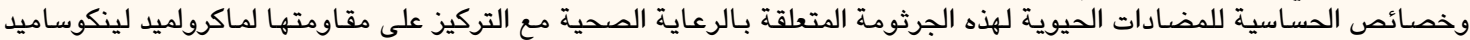

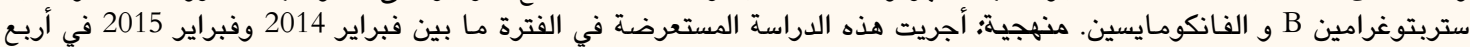

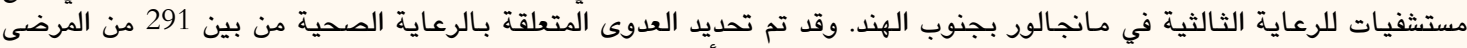

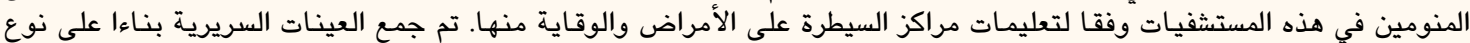

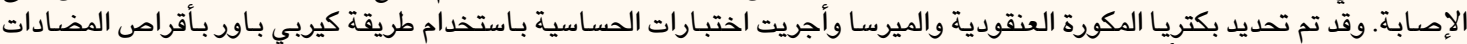

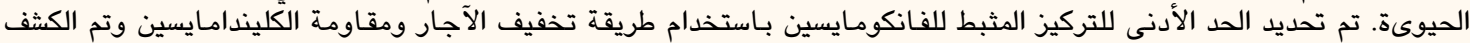

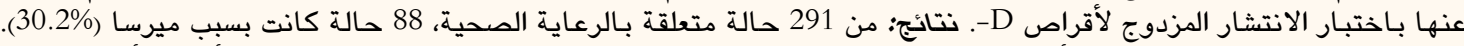

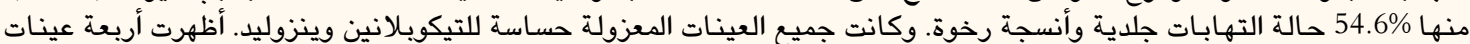

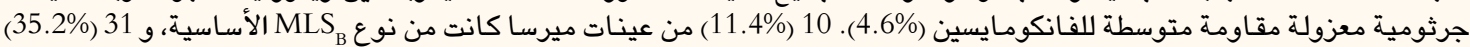

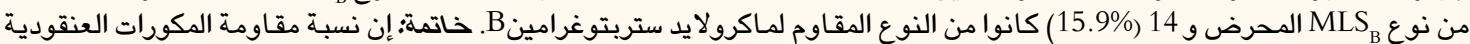

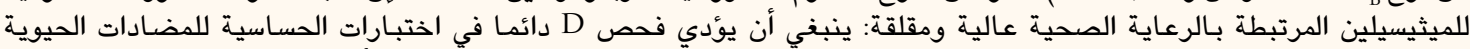

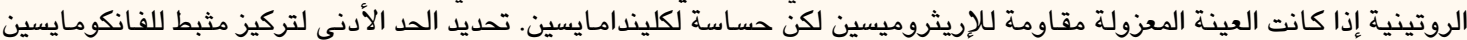

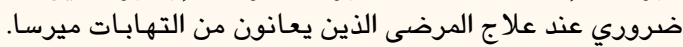

كلمات مفتاحية: العدوى المرتبطة بالرعاية الصحية؛ مقاومة المضادات الحيوية؛ المكورات العنقودية الذهبية الدقاومة للميثيسيلين؛ الظواهر؛ 


\begin{abstract}
Advances in KNOWledge
A high rate of inducible macrolide-lincosamide-streptogramin $B\left(M L S_{B}\right)$ phenotypes were found among healthcare-associated methicillin-resistant Staphylococcus aureus (MRSA) isolates identified from clinical specimens in the current study.

Four cases of healthcare-associated MRSA with intermediate resistance to vancomycin were detected. This finding challenges the efficacy of vancomycin in cases of serious multidrug-resistant healthcare-associated MRSA infections.

\section{Application to Patient Care}

The results of this study indicate the necessity of performing double-disk diffusion tests to identify inducible $M L S_{B}$ resistance to prevent the therapeutic failure of clindamycin in healthcare-associated MRSA cases.

Additionally, these findings suggest that the minimum inhibitory concentration of vancomycin should be determined in order to guide therapy.
\end{abstract}

$\mathrm{M}$ ETHICILLIN-RESISTANT STAPHYLOCOCCUS aureus (MRSA) was first identified in the 1960s and continues to be a significant pathogen worldwide, causing a variety of infections from minor skin and soft tissue infections to severe osteomyelitis, bacteraemia and sepsis. ${ }^{1}$ Healthcareassociated MRSA spreads through direct or indirect contact and normally exhibits multidrug resistance. ${ }^{1}$ The distribution of healthcare-associated MRSA varies geographically; rates in the Western Pacific region, Latin America, the USA and Europe are 46.0\%, 34.9\%, $34.2 \%$ and $26.3 \%$, respectively. ${ }^{2}$ In contrast, northern European countries such as Denmark, the Netherlands, Iceland and Sweden have reported rates of $<1.0 \%{ }^{3}$ In Asia, the prevalence varies from $22.6-86.5 \%$, with the highest rate reported in Sri Lanka and the lowest in India. ${ }^{4}$ Nevertheless, the rate of healthcare-associated MRSA in India has steadily increased over the past few years, from $<20.0 \%$ in 2002 to $39.6 \%$ in $2012 .^{5-8}$

The rise of multidrug-resistant healthcareassociated MRSA cases has reduced the number of available therapeutic options for these infections. Potential antibiotics include daptomycin, linezolid, quinupristin/dalfopristin, trimethoprim/sulfamethoxazole and vancomycin. ${ }^{9}$ Glycopeptides, in particular vancomycin, are the treatment of choice for serious infections. ${ }^{9}$ The macrolide-lincosamide-streptogramin $\mathrm{B}\left(\mathrm{MLS}_{\mathrm{B}}\right)$ group of antibiotics can be used to treat less severe skin and soft tissue infections. ${ }^{9}$ The lincosamide antibiotic clindamycin is often preferred because of its low cost and pharmacokinetic properties, including significant tissue penetration, an extended half-life and the ability to inhibit toxin production. ${ }^{9,10}$ Unfortunately, the widespread use of $\mathrm{MLS}_{\mathrm{B}}$ antibiotics has resulted in increased resistance which occurs either due to an efflux mechanism encoded by the methionine sulfoxide reductase $A$ gene or a ribosomal target site modification encoded by the erythromycin ribosomal methylase gene. ${ }^{10}$ Constitutive $\mathrm{MLS}_{\mathrm{B}}\left(\mathrm{CMLS}_{\mathrm{B}}\right)$ resistance can be detected in routine susceptibility testing by its resistance to both erythromycin and clindamycin; in contrast, bacteria with inducible $\mathrm{MLS}_{\mathrm{B}}\left(\mathrm{iMLS}_{\mathrm{B}}\right)$ resistance appear resistant to erythromycin but susceptible to clindamycin in routine testing, thereby posing a problem in detection. ${ }^{10}$ As a result, if clindamycin is used for the treatment of infections caused by these strains, the treatment will fail. It is therefore important to detect this kind of resistance.

The available literature does not reveal precise data on $\mathrm{MLS}_{\mathrm{B}}$-resistant phenotypes and the susceptibility profile to vancomycin among healthcare-associated MRSA-affected populations from South India. Consequently, the present study was conducted to determine the clinical characteristics and antibiotic susceptibility patterns of MRSA isolates from clinical specimens taken from a South Indian cohort, with emphasis on the aforementioned factors.

\section{Methods}

This cross-sectional study was conducted from February 2014 to February 2015 among four tertiary care hospitals in Mangalore, South India. Two of the hospitals were governmental hospitals with 600 and 250 beds, respectively, while the other two were private hospitals with 510 and 251 beds, respectively. The governmental hospitals had general surgery and neurosurgical departments, burn units, general intensive care units (ICUs) and urology, nephrology, dermatology, gynaecology and paediatric wards. In addition to the aforementioned departments, the private hospitals also had oncology, haematology, cardiothoracic and nephrosurgery departments as well as general, nephrosurgery, neurosurgery and cardiothoracic ICUs.

The inclusion criteria included inpatients presenting to one of the four hospitals with localised or systemic conditions resulting from the presence of an infectious agent or its toxins which was not present or incubating at the time of admission to the hospital but only became evident 48 hours after admission or longer. In addition, patients with a history of hospitalisation, haemodialysis, surgery or admission to a long-term healthcare facility within the previous 
year and those with indwelling catheters, intravenous lines or other percutaneous medical devices at the time of culture collection were included. Outpatients and those with no healthcare-associated MRSA risk factors who were diagnosed with an S. aureus infection within 48 hours of hospitalisation were excluded.11,12 The sample size was calculated using the following formula: ${ }^{13}$

$$
n=\frac{Z_{1-\alpha}^{2} p q}{d^{2}}
$$

where $Z_{1-\alpha}$ is 1.96 ( $95 \%$ confidence interval), $\mathrm{p}$ is 0.37 (expected proportion), $\mathrm{q}$ is 0.63 (1-p) and $\mathrm{d}$ is 0.0555 (relative precision of $15 \%$ of $\mathrm{p}$, i.e. allowable error). The expected proportion was selected based on a previous study. ${ }^{14}$ The total sample size required was therefore 291 patients.

The criteria of the Centers for Disease Control and Prevention were used to identify healthcare-associated infections due to $S$. aureus. ${ }^{12}$ A structured pro forma was used to collect the demographic and clinical details of the patients. Based on the type and site of infection, clinical specimens such as pus/exudates, blood, sput$u m$ and indwelling medical devices (i.e. central line tips, peripheral intravenous catheters, endotracheal tubes and urinary catheters) were collected from the patients and processed by the Department of Microbiology at the Kasturba Medical College in Mangalore. Gram staining of the specimens was performed followed by blood agar and MacConkey's agar cultures using standard bacteriological procedures. ${ }^{15}$ S. aureus bacteria were identified by colony morphology, Gram staining and catalase, slide and tube coagulase and deoxyribonuclease tests. ${ }^{15}$ The cefoxitin $(30.00 \mu \mathrm{g})$ disk diffusion method was used to detect methicillin resistance in S. aureus isolates. ${ }^{16}$

Antibiotic susceptibility was tested using the Kirby-Bauer disk diffusion method and the results interpreted using the guidelines of the Clinical and Laboratory Standards Institute (CLSI). ${ }^{16}$ The following antibiotics were tested: ciprofloxacin (5.00 $\mu \mathrm{g})$, clindamycin $(2.00 \mu \mathrm{g})$, trimethoprim/ sulfamethoxazole $(1.25 \mu \mathrm{g} / 23.75 \mu \mathrm{g})$, erythromycin $(15.00 \mu \mathrm{g})$, gentamicin $(10.00 \mu \mathrm{g})$, linezolid $(30.00 \mu \mathrm{g})$, penicillin $(10 \mathrm{U})$, rifampicin $(5.00 \mu \mathrm{g})$ and teicoplanin $(30.00 \mu \mathrm{g})$. All antibiotics were purchased from HiMedia Laboratories Ltd. (Mumbai, Maharashtra, India). An S. aureus strain (ATCC ${ }^{\circledR} 25923^{\mathrm{TM}}$, American Type Culture Collection, Manassas, Virginia, USA) was used as the control.

The minimum inhibitory concentration (MIC) of vancomycin was determined by the agar dilution method using CLSI guidelines. ${ }^{17}$ Gradient plates of Mueller-Hinton agar were prepared with vancomycin
(0.062-128.000 $\mu \mathrm{g} / \mathrm{mL})$. Three or four MRSA colonies grown on blood agar were picked and inoculated in Mueller-Hinton broth (MHB) and incubated at $35{ }^{\circ} \mathrm{C}$ for $4-6$ hours. The turbidity of the broth culture was matched with a 0.5 McFarland standard (bacterial count $\approx 1.5 \times 10^{8}$ colony-forming unit $[\mathrm{cfu}] / \mathrm{mL}$ ) and then diluted at a ratio of 1:10 in sterile MHB to ensure a concentration of $1.5 \times 10^{7} \mathrm{cfu} / \mathrm{mL}$. From this culture, $2 \mu \mathrm{L}$ of the suspension was spotinoculated on each plate and incubated at $35{ }^{\circ} \mathrm{C}$ for 24 hours. MRSA strains with MICs of $\leq 2.00 \mu \mathrm{g} / \mathrm{mL}$, $4.00-8.00 \mu \mathrm{g} / \mathrm{mL}$ and $\geq 16.00 \mu \mathrm{g} / \mathrm{mL}$ were considered susceptible, intermediate and resistant to vancomycin, respectively. ${ }^{16}$ S. aureus (ATCC ${ }^{\circledR} 29213^{\mathrm{TM}}$, American Type Culture Collection) and Enterococcus faecalis (ATCC $^{\circledR} 29212^{\mathrm{TM}}$, American Type Culture Collection) strains were used as vancomycin-susceptible controls. Another E. faecalis strain (ATCC ${ }^{\circledR} 51299^{\mathrm{TM}}$, American Type Culture Collection) was used as the vancomycinresistant control.

The $\mathrm{MLS}_{\mathrm{B}}$ phenotypes were identified as described previously. ${ }^{16,18}$ MRSA strains resistant to both erythromycin and clindamycin in routine antibiotic susceptibility testing were considered $\mathrm{CMLS}_{\mathrm{B}}$ phenotypes. A double-disk diffusion test (D-test) was performed to determine MRSA strains which were resistant to erythromycin but sensitive to clindamycin (iMLS ${ }_{\mathrm{B}}$ phenotypes). Erythromycin $(15.00 \mu \mathrm{g})$ and clindamycin $(2.00 \mu \mathrm{g})$ disks were placed on a MuellerHinton agar plate containing a lawn culture of the test isolate at a distance of $15 \mathrm{~mm}$ edge to edge. The plate was incubated at $35{ }^{\circ} \mathrm{C}$ for $16-18$ hours. Any flattening of the zone of inhibition (D-shape) around the clindamycin disk adjacent to the erythromycin disk was subsequently observed; strains with a D-shaped zone of inhibition were considered iMLS $_{B}$ phenotypes. Strains of MRSA which were resistant to erythromycin, susceptible to clindamycin and were also D-test negative (had no D-shaped zone of inhibition) were considered macrolide-streptogramin $\mathrm{B}\left(\mathrm{MS}_{\mathrm{B}}\right)$ phenotypes.

The Statistical Package for the Social Sciences (SPSS), Version 16.0 (IBM Corp., Chicago, Illinois, USA) was used to analyse the data. Rates of MRSA infections were presented as percentages. The Pearson's Chi-squared test was used to analyse results between the two groups of categorical variables. A $P$ value of $\leq 0.050$ was considered statistically significant.

This study was approved by the Institutional Ethics Committee of Kasturba Medical College (\#IECKMCMLR01-14/14). Informed consent was collected from all patients prior to their participation in the study. 
Table 1: Type of healthcare-associated methicillinresistant Staphylococcus aureus and methicillinsensitive Staphylococcus aureus infections $(\mathrm{N}=291)$

\begin{tabular}{lccc} 
Type of infection & \multicolumn{2}{c}{ n (\%) } & $\begin{array}{c}\boldsymbol{P} \\
\text { value* }\end{array}$ \\
& $\begin{array}{c}\text { MRSA } \\
(\mathbf{n}=\mathbf{8 8})\end{array}$ & $\begin{array}{c}\text { MSSA } \\
(\mathbf{n}=\mathbf{2 0 3})\end{array}$ & \\
Surgical site & $18(20.5)$ & $77(37.9)$ & $0.004+$ \\
Cellulitis & $17(19.3)$ & $25(12.3)$ & 0.118 \\
Bed sore & $11(12.5)$ & $16(7.9)$ & 0.212 \\
Catheter-associated & $14(15.9)$ & $3(1.5)$ & $<0.001^{\dagger}$ \\
bacteraemia & & & \\
Ulcer & $8(9.1)$ & $23(11.3)$ & 0.569 \\
Burn wound & $8(9.1)$ & $28(13.8)$ & 0.263 \\
TEN & $3(3.4)$ & $5(2.5)$ & 0.665 \\
Bacteraemia & $6(6.8)$ & $2(1.0)$ & $0.005^{\dagger}$ \\
Psoas abscess & $1(1.1)$ & $0(0.0)$ & 0.665 \\
Empyema & $1(1.1)$ & $0(0.0)$ & 0.665 \\
Pneumonia & $0(0.0)$ & $8(3.9)$ & 0.134 \\
Osteomyelitis & $1(1.1)$ & $14(6.9)$ & 0.041 \\
Catheter-associated UTI & $0(0.0)$ & $2(1.0)$ & 0.350
\end{tabular}

TEN = toxic epidermal necrolysis; $M R S A=$ methicillin-resistant Staphylococcus aureus; MSSA = methicillin-sensitive Staphylococcus aureus; $U T I=$ urinary tract infection

"Determined using the Chi-squared test. ${ }^{\dagger}$ Statistically significant at $P \leq 0.050$.

\section{Results}

A total of 291 non-repetitive healthcare-associated-S. aureus cases were isolated during the study period; 119 and 12 cases were from the two governmental hospitals and 95 and 65 cases were from the two private hospitals, respectively. The rates of healthcareassociated MRSA in the two government hospitals and two private hospitals were $41.2 \%, 33.3 \%, 22.1 \%$ and $21.5 \%$, respectively. No statistically significant differences were observed between male and female patients. Patients aged $31-40$ and $41-50$ years old were most likely to beinfected with MRSA. The different types of $S$. aureus infections found are shown in Table 1 . Of the S. aureus cases, 95 (32.7\%) were isolated from surgical site infections, 42 (14.4\%) from cellulitis cases, 25 (8.6\%) from blood stream infections (including bacteraemia and catheter-associated bacteraemia infections) and 15 (5.2\%) from osteomyelitis cases. A total of 88 S. aureus cases (30.2\%) were methicillinresistant. Of the MRSA cases, $54.6 \%$ were skin and soft tissue infections, comprising cellulitis, bed sore, ulcer, burn wound, toxic epidermal necrolysis and psoas abscess infections. MRSA was significantly more frequent in bacteraemia infections while methicillin-
Table 2: Antibiotic resistance patterns of healthcareassociated methicillin-resistant Staphylococcus aureus and methicillin-sensitive Staphylococcus aureus isolates $(\mathrm{N}=291)$

\begin{tabular}{|c|c|c|c|}
\hline \multirow[t]{2}{*}{ Antibiotic } & \multicolumn{2}{|c|}{ n (\%) } & \multirow[t]{2}{*}{$P$ value } \\
\hline & $\begin{array}{c}\text { Resistant } \\
\text { MRSA } \\
\text { isolates } \\
(\mathrm{n}=\mathbf{8 8})\end{array}$ & $\begin{array}{c}\text { Resistant } \\
\text { MSSA } \\
\text { isolates } \\
(\mathbf{n}=203)\end{array}$ & \\
\hline Ciprofloxacin & $66(75.0)$ & $68(33.5)$ & $<0.001^{\dagger}$ \\
\hline Clindamycin & $45(51.1)$ & 33 (16.3) & $<0.001^{\dagger}$ \\
\hline $\begin{array}{l}\text { Trimethoprim/ } \\
\text { sulfamethoxazole }\end{array}$ & $52(59.1)$ & $59(29.1)$ & $<0.001^{\dagger}$ \\
\hline Erythromycin & $55(62.5)$ & $64(31.5)$ & $<0.001^{\dagger}$ \\
\hline Gentamicin & $37(42.1)$ & $22(10.8)$ & $<0.001^{\dagger}$ \\
\hline Linezolid & $0(0.0)$ & $0(0.0)$ & - \\
\hline Penicillin & $88(100.0)$ & $181(89.2)$ & $0.001^{\dagger}$ \\
\hline Rifampicin & $1(1.1)$ & $0(0.0)$ & 0.128 \\
\hline Teicoplanin & $0(0.0)$ & $0(0.0)$ & - \\
\hline Tetracycline & $33(37.5)$ & $37(18.2)$ & $<0.001^{\dagger}$ \\
\hline $\begin{array}{l}S S A=\text { methicillin }- \\
S A=\text { methicillin }\end{array}$ & $\begin{array}{l}\text { unt Staph } \\
\text { ve Staphy }\end{array}$ & $\begin{array}{l}\text { us aureus; } \\
\text { us aureus. }\end{array}$ & \\
\hline
\end{tabular}

sensitive $S$. aureus (MSSA) was significantly more frequent in surgical site infections $(P<0.005)$.

The antibiotic resistance patterns of the MRSA and MSSA isolates are shown in Table 2. All of the MRSA and MSSA isolates were susceptible to teicoplanin and linezolid, while the majority of MRSA isolates were resistant to ciprofloxacin $(75.0 \%)$, clindamycin (51.1\%), trimethoprim/sulfamethoxazole (59.1\%) and erythromycin (62.5\%). In comparison to MSSA isolates, MRSA isolates were significantly more resistant to ciprofloxacin, clindamycin, trimethoprim/ sulfamethoxazole, erythromycin, gentamicin and tetracycline $(P<0.005)$.

Of the MRSA strains, 84 (95.5\%) were susceptible to vancomycin while four (4.6\%) demonstrated intermediate resistance. None of the strains were resistant to vancomycin. The $\mathrm{MIC}_{90}$ and $\mathrm{MIC}_{50}$ values of vancomycin were $2.00 \mu \mathrm{g} / \mathrm{mL}$ and $1.00 \mu \mathrm{g} / \mathrm{mL}$, respectively. The four MRSA strains with intermediate resistance to vancomycin were isolated from four patients with osteomyelitis, an infected wound, a psoas abscess and catheter-associated bacteraemia, respectively; three of the isolates were from patients in a governmental hospital and the remaining isolate was from a patient in a private hospital. Two of the isolates with intermediate resistance to vancomycin were of the $\mathrm{CMLS}_{\mathrm{B}}$ phenotype and two were of the 
Table 3: Macrolide-lincosamide-streptogramin B phenotypes among patients with healthcare-associated methicillin-resistant Staphylococcus aureus infections $(\mathrm{N}=88)$

\begin{tabular}{lccc} 
Hospital & \multicolumn{3}{c}{$\mathbf{n}(\%)$} \\
& $\begin{array}{c}\mathrm{cMLS}_{\mathrm{B}} \\
\text { phenotype }\end{array}$ & $\begin{array}{c}\text { iMLS } \\
\text { phenotype }\end{array}$ & $\begin{array}{c}\mathrm{MS}_{\mathrm{B}} \\
\text { phenotype }\end{array}$ \\
$\begin{array}{l}\text { Government } \\
\text { hospital 1 }\end{array}$ & $6(6.8)$ & $23(26.1)$ & $8(9.1)$ \\
$\begin{array}{l}\text { Government } \\
\text { hospital 2 }\end{array}$ & $1(1.1)$ & $1(1.1)$ & $0(0.0)$ \\
$\begin{array}{l}\text { Private hospital 1 } \\
\text { Private hospital 2 }\end{array}$ & $1(1.1)$ & $4(4.5)$ & $3(3.4)$ \\
Total $^{*}$ & $\mathbf{1 0 ( 1 1 . 4 )}$ & $31(35.2)$ & $\mathbf{1 4}(15.9)$
\end{tabular}

$M L S_{B}=$ macrolide-lincosamide-streptogramin $B ; c M L S_{B}=$ constitutive $M L S_{B} ; i M L S_{B}=$ inducible $M L S_{B} ; M S_{B}=$ macrolide-streptogramin $B$. *The remaining 33 isolates were sensitive to both clindamycin and erythromycin.

$\mathrm{MS}_{\text {в }}$ phenotype. A total of 10 MRSA isolates (11.4\%) were resistant to both erythromycin and clindamycin (cMLS ${ }_{\mathrm{B}}$ phenotype), while 45 (51.1\%) were resistant to erythromycin but susceptible to clindamycin. Of these 45 strains, $31(68.9 \%)$ were D-test positive (iMLS $_{B}$ phenotype) and 14 (31.1\%) were D-test negative $\left(\mathrm{MS}_{\mathrm{B}}\right.$ phenotype). The remaining 33 MRSA isolates (37.5\%) were sensitive to both clindamycin and erythromycin. The $\mathrm{MLS}_{\mathrm{B}}$ phenotypes of the MRSA isolates from the four hospitals are shown in Table 3.

\section{Discussion}

Considerable geographical variation exists in the prevalence of healthcare-associated MRSA infections. ${ }^{2-4}$ The present study revealed a high cumulative rate of health-associated MRSA infections among patients admitted to four tertiary care hospitals in Mangalore. However, the rate was lower than the $41 \%$ national prevalence rate reported by the Indian Network for Surveillance of Antimicrobial Resistance (INSAR) Group based on data collected from 15 tertiary centres. ${ }^{8}$ Nevertheless, the rate in the present study was higher than that that reported from the same four hospitals in a previous study in 2011 (25.25\%). ${ }^{7}$ Akoğlu et al. reported a higher rate of healthcare-associated MRSA in Turkey (71.5\%) while the Australian Group on Antimicrobial Resistance reported a similar rate (30.3\%) to that of the current study. ${ }^{19,20}$ However, it is important to note that the current study included patients with potential predisposing risk factors to healthcare-associated MRSA infections, including previous surgeries/trauma, the presence of indwelling devices such as catheters and lengthy hospital stays. ${ }^{12}$ In terms of gender predilection, no significant difference was found between male and female patients in the current study; this observation is in agreement with previous research. ${ }^{21}$

A significantly greater number of healthcareassociated MRSA isolates in the current study were resistant to non- $\beta$-lactam antibiotics in comparison with healthcare-associated MSSA isolates. A similar observation was made by the INSAR Group. ${ }^{8}$ This could be due to the unique molecular properties of MRSA bacteria-these strains carry the staphylococcal cassette chromosome mec (SCCmec), a mobile genetic element which includes the $m e c A$ gene that codes for methicillin resistance. ${ }^{22}$ Healthcare-associated MRSA carries SCCmec types I, II or III; these elements are relatively large and are associated with resistance to many other non- $\beta$-lactam antibiotics. ${ }^{22}$ This kind of multidrug resistance is challenging for clinicians when selecting an appropriate antibiotic for treatment.

Among different phenotypes of $\mathrm{MLS}_{\mathrm{B}}$ resistance, detection of $\mathrm{CMLS}_{\mathrm{B}}$ does not pose a problem because these strains are resistant to both erythromycin and clindamycin in routine susceptibility testing; however, the detection of $\mathrm{iMLS}_{\mathrm{B}}$ and $\mathrm{MS}_{\mathrm{B}}$ phenotypes is not possible with standard methods because these strains appear resistant to erythromycin but susceptible to clindamycin. ${ }^{10}$ Hence, clindamycin treatment will fail with iMLS ${ }_{B}$ MRSA strains. ${ }^{10}$ Conversely, if clindamycin is not considered as a treatment option due to the suspicion that the strain might be resistant, patients with clindamycin-susceptible infections would be deprived of the correct antibiotic. In the present study, more than a third of the MRSA isolates were of the $\mathrm{iMLS}_{\mathrm{B}}$ phenotype; this finding is higher than those reported from previous Indian studies. ${ }^{7,23}$ Studies from other parts of the world have shown iMLS ${ }_{B}$ phenotype rates ranging from $7.1-56 \%{ }^{24-26}$ These results clearly support the use of a D-test to identify healthcareassociated MRSA strains with $\mathrm{iMLS}_{\mathrm{B}}$ resistance.

Vancomycin is considered an appropriate antibiotic for the treatment of serious MRSA infections and its use has increased dramatically over the past 20 years due to the emergence of multidrug-resistant healthcare-associated MRSA.9,27 However, the recent emergence of vancomycin-intermediate $S$. aureus (VISA) and vancomycin-resistant $S$. aureus (VRSA) infections has added to the difficulty in selecting the appropriate antibiotic for these bacteria. ${ }^{1}$ Several reports of MRSA infections with reduced susceptibility to vancomycin have been reported from France, Japan, Korea, Germany and the USA. ${ }^{27}$ While VRSA infections are not yet a problem in India, VISA strains have been reported from some centres. ${ }^{28,29}$ There were four VISA cases observed in the present study; however, routine testing does not differentiate between VISA and vancomycin-susceptible strains. ${ }^{16,17}$ In the 
present study, it was observed that agar dilution was useful in determining the vancomycin MIC and also contributed to differentiating VISA from vancomycinsusceptible strains. Tandel et al. have previously shown that the Etest ${ }^{\circledR}$ (bioMérieux, Marcy-l'Étoile, Lyon, France) can also be used for this purpose. ${ }^{30}$ Determining the MIC of vancomycin is required before selecting this antibiotic for the treatment of a healthcare-associated MRSA infection. Fortunately, all MRSA strains were susceptible to linezolid, leaving it as the choice of treatment in these cases.

Molecular testing was not performed on the MRSA isolates and was therefore a limitation of the present study. There is an urgent need for further studies on the judicious use of vancomycin and the surveillance of antibiotic resistance. In addition, strategies need to be implemented in order to prevent healthcareassociated infections in India.

\section{Conclusion}

A high rate of healthcare-associated MRSA infections was observed, indicating that this type of infection is a significant concern for the four hospitals included in this study. A greater number of MRSA cases were multidrug-resistant as compared with MSSA. A D-test should be performed before selecting clindamycin to treat cases of MRSA that appear susceptible to clindamycin but are resistant to erythromycin according to standard antibiotic susceptibility testing. The MIC of vancomycin should be determined and susceptibility should be proven before considering this antibiotic for the treatment of serious MRSA infections.

\section{CONFLICT OF INTEREST}

The authors declare no conflicts of interest.

\section{References}

1. DeLeo FR, Chambers HF. Reemergence of antibiotic-resistant Staphylococcus aureus in the genomics era. J Clin Invest 2009; 119:2464-74. doi: 10.1172/JCI38226.

2. Diekema DJ, Pfaller MA, SchmitzFJ, SmayevskyJ, BellJ, Jones RN, et al. Survey of infections due to Staphylococcus species: Frequency of occurrence and antimicrobial susceptibility of isolates collected in the United States, Canada, Latin America, Europe, and the Western Pacific region for the SENTRY Antimicrobial Surveillance Program, 1997-1999. Clin Infect Dis 2001; 32:S114-32. doi: 10.1086/320184.

3. Tiemersma EW, Bronzwaer SL, Lyytikäinen O, Degener JE, Schrijnemakers P, Bruinsma N, et al. Methicillin-resistant Staphylococcus aureus in Europe, 1999-2002. Emerg Infect Dis 2004; 10:1627-34.
4. Song JH, Hsueh PR, Chung DR, Ko KS, Kang CI, Peck KR, et al. Spread of methicillin-resistant Staphylococcus aureus between the community and the hospitals in Asian countries: An ANSORP study. J Antimicrob Chemother 2011; 66:1061-9. doi: $10.1093 / \mathrm{jac} / \mathrm{dkr} 024$.

5. Tahnkiwale SS, Roy S, Jalgaonkar SV. Methicillin resistance among isolates of Staphylococcus aureus: Antibiotic sensitivity pattern \& phage typing. Indian J Med Sci 2002; 56:330-4.

6. Bouchiat C, El-Zeenni N, Chakrakodi B, Nagaraj S, Arakere G, Etienne J. Epidemiology of Staphylococcus aureus in Bangalore, India: Emergence of the ST217 clone and high rate of resistance to erythromycin and ciprofloxacin in the community. New Microbes New Infect 2015; 7:15-20. doi: 10.1016/j. nmni.2015.05.003.

7. Sharma NK, Garg R, Baliga S, Bhat KG. Nosocomial infections and drug susceptibility patterns in methicillin sensitive and methicillin resistant Staphylococcus aureus. J Clin Diagn Res 2013; 7:2178-80. doi: 10.7860/JCDR/2013/6750.3463.

8. Indian Network for Surveillance of Antimicrobial Resistance (INSAR) Group, India. Methicillin resistant Staphylococcus aureus (MRSA) in India: Prevalence \& susceptibility pattern. Indian J Med Res 2013; 137:363-9.

9. Liu C, Bayer A, Cosgrove SE, Daum RS, Fridkin SK, Gorwitz RJ, et al. Clinical practice guidelines by the Infectious Diseases Society of America for the treatment of methicillin-resistant Staphylococcus aureus infections in adults and children: Executive summary. Clin Infect Dis 2011; 52:285-92. doi: $10.1093 / \mathrm{cid} / \mathrm{cir} 034$

10. Leclercq R. Mechanism of resistance to macrolides and lincosamides: Nature of the resistant elements and their clinical implications. Clin Infect Dis 2002; 34:482-92. doi: $10.1086 / 324626$

11. David MZ, Daum RS. Community-associated methicillinresistant Staphylococcus aureus: Epidemiology and clinical consequences of an emerging epidemic. Clin Microbiol Rev 2010; 23:616-87. doi: 10.1128/CMR.00081-09.

12. Centers for Disease Control and Prevention. CDC/NHSN surveillance definition for specific types of infection. From: www.cdc.gov/nhsn/PDFs/pscManual/17pscNosInfDef_ current.pdf Accessed: Feb 2016

13. Mahajan BK. Methods in Biostatistics: For Medical Student and Research Workers, 8th ed. New Delhi, India: Jaypee Brothers Medical Publishers Ltd., 2016. Pp. 113-27. doi: 10.50 05/jp/books/12646.

14. Rashid A, Rashid H, Qureshi UR, Rashid A. Conquering methicillin resistant Staphylococcus aureus: Prespectives and treatment options. Int Arch Med 2015; 8:1-7. doi: $10.3823 / 1666$.

15. Bannerman TL. Staphylococci, Micrococcus, and other catalase positive cocci that grow aerobically. In: Murray PR, Baron EJ, Jorgenson JH, Pfaller MA, Yolken RH (Eds). Manual of Clinical Microbiology, 8th ed. Washington DC, USA: ASM Press, 2003. Pp. 384-404.

16. Clinical and Laboratory Standards Institute. M100-S25: Performance standards for antimicrobial susceptibility testing Twenty-fifth informational supplement. From: http://shop.clsi. org/microbiology-documents/ Accessed: Feb 2016.

17. Clinical and Laboratory Standards Institute. M100-S22 Performance standards for antimicrobial susceptibility testing - Twenty-second informational supplement. From: http://shop. clsi.org/microbiology-documents/ Accessed: Feb 2016.

18. Steward CD, Raney PM, Morrell AK, Williams PP, McDougal LK Jevitt L, et al. Testing for induction of clindamycin resistance in erythromycin-resistant isolates of Staphylococcus aureus. J Clin Microbiol 2005; 43:1716-21. doi: 10.1128/JCM.43.4.17161721.2005 
19. Akoğlu H, Zarakolu P, Altun B, Ünal S. [Epidemiological and molecular characteristics of hospital-acquired methicillinresistant Staphylococcus aureus strains isolated in Hacettepe University Adult Hospital in 2004-2005.] Mikrobiyol Bul 2010; 44:343-55.

20. Coombs GW, Nimmo GR, Pearson JC, Collignon PJ, Bell JM, McLaws ML, et al.; Australian Group on Antimicrobial Resistance. Australian Group on Antimicrobial Resistance Hospital-onset Staphylococcus aureus Surveillance Programme annual report, 2011. Commun Dis Intell Q Rep 2013; 37:E210-18.

21. Abbas A, Nirwan PS, Srivastava P. Prevalence and antibiogram of hospital acquired-methicillin resistant Staphylococcus aureus and community acquired-methicillin resistant Staphylococcus aureus at a tertiary care hospital National Institute of Medical Sciences. Community Acquir Infect 2015 2:13-15. doi: 10.4103/2225-6482.153857.

22. Hiramatsu K, Katayama Y, Yuzawa H, Ito T. Molecular genetics of methicillin-resistant Staphylococcus aureus. Int J Med Microbiol 2002; 292:67-74. doi: 10.1078/1438-4221-00192.

23. Chadha T, Kulsum SN, Adlekha S, Mailapur PC. Comparison of antibiotic susceptibility pattern of community- and hospitalassociated methicillin-resistant Staphylococcus aureus with special reference to inducible clindamycin resistance in a tertiary care hospital in southern India. Med J D Y Patil Univ 2014; 7:439-42. doi: 10.4103/0975-2870.135257.

24. Schmitz FJ, Sadurski R, Kray A, Boos M, Geisel R, Köhrer K, et al. Prevalence of macrolide-resistance genes in Staphylococcus aureus and Enterococcus faecium isolates from 24 European university hospitals. J Antimicrob Chemother 2000; 45:891-4. doi: 10.1093/jac/45.6.891.
25. Schreckenberger PC, Ilendo $\mathrm{E}$, Ristow KL. Incidence of constitutive and inducible clindamycin resistance in Staphylococcus aureus and coagulase-negative staphylococci in a community and a tertiary care hospital. J Clin Microbiol 2004; 42:2777-9. doi: 10.1128/JCM.42.6.2777-2779.2004.

26. Patel M, Waites KB, Moser SA, Cloud GA, Hoesley CJ. Prevalence of inducible clindamycin resistance among community- and hospital- associated Staphylococcus aureus isolates. J Clin Microbiol 2006; 44:2481-4. doi: 10.1128/ JCM.02582-05.

27. Loomba PS, Tanjea J, Mishra B. Methicillin and vancomycin resistant S. aureus in hospitalized patients. J Glob Infect Dis 2010; 2:275-83. doi: 10.4103/0974-777X.68535.

28. Thati V, Shivannavar CT, Gaddad SM. Vancomycin resistance among methicillin resistant Staphylococcus aureus isolated from intensive care units of tertiary care hospitals in Hyderabad. Indian J Med Res 2011; 134:704-8. doi: 10.4103/0971-5916. 91001.

29. Dhawan B, Gadepalli R, Rao C, Kapil A, Sreenivas V. Decreased susceptibility to vancomycin in methicillin-resistant Staphylococcus aureus: A 5 year study in an Indian tertiary hospital. J Med Microbiol 2010; 59:375-6. doi: 10.1099/jmm. 0.011940-0.

30. Tandel K, Praharaj AK, Kumar S. Differences in vancomycin MIC among MRSA isolates by agar dilution and $\mathrm{E}$ test method. Indian J Med Microbiol 2012; 30:453-5. doi: 10.4103/02550857.103768. 